\title{
A Blockchain-Based Architecture for Smart Healthcare System: A Case Study of Saudi Arabia
}

\author{
Saleh Albahli ${ }^{*}$, Rehan Ullah Khan ${ }^{1}$, Ali Mustafa Qamar ${ }^{2}$ \\ ${ }^{1}$ Department of Information Technology, College of Computer, Qassim University, Saudi Arabia \\ ${ }^{2}$ Department of Computer Science, College of Computer, Qassim University, Saudi Arabia
}

\author{
A R T I C L E I N F O \\ Article history: \\ Keywords: \\ Healthcare \\ Blockchain \\ Smart healthcare \\ Smart cities \\ Healthcare cloud
}

Received: 09 October, 2019

Accepted: 04 January, 2020

Online: 15 January, 2020

\begin{abstract}
A B S T R A C T
eHealth is the use of Information and Communication Technologies (ICT) to enhance health quality access. Health care innovations are an essential element of Vision 2030 and National Transformation Program (NTP) 2020 operational plan that will lead to an improvement in the quality of health care in Saudi Arabia. The objective of this paper is to address the healthcare system limitations in the Kingdom of Saudi Arabia in general and the Qassim region in particular to global standards. In practice, the creation of an infrastructure for storing and seamless sharing of health data between different entities is studied. Furthermore, an in-depth analysis of current practices w.r.t. the health data, as well as finding similarities between NTP 2020 and Health 2020 (European Union) has been performed. A multi-level blockchain eHealth system is proposed to provide seamless electronic health records to the patients. Moreover, the current practices being employed in Qassim province, KSA has been analyzed. It was found that private hospitals give access to medical reports to their patients besides allowing them to manage their appointments. On the other hand, access to the government hospitals' medical records is minimal.
\end{abstract}

\section{Introduction}

The thrust of technology has provided a lot of facilities for everyday life. The technology transformation in the medical domain evolves within the political, administrative and socioeconomic factors of a particular country. Also, the technology introduced by using various platforms including e-Health, Telemedicine, and $\mathrm{m}$-Health provides the facility to disseminate health-related information distributed among clinics, health care providers and patients. e-Health is a subset of telemedicine and $\mathrm{m}$ Health [1-3]. The importance of these state-of-the-art innovative approaches towards improved health care systems and quality of life in the Kingdom of Saudi Arabia (KSA) is highlighted through a number of project calls initiated by the health ministry. Also, the KSA government stresses that the country's healthcare institutions must work as an allocative and collaborative body for the wellbeing of citizens.

In the Middle East, the KSA healthcare sector is the largest. According to the 2018 budget, the KSA spent $36 \%$ of its budget on the health sector which holds the largest share. Population

*Saleh Albahli, Email: salbahli@qu.edu.sa growth, emerging diseases, and fluctuating international scenarios are pushing the up-gradation of the KSA health system. The government is also taking steps towards marketization and privatization as a core policy of health system reforms. With the up-gradation of traditional health to e-Health, the privacy and security of patients' data is also a significant concern [4].

The current Saudi Arabian government has initiated a Vision 2030 [5]. The Vision 2030 is a bold but achievable blueprint for an ambitious nation. For the capacity building and for achieving Vision 2030 objectives, the KSA government has launched the National Transformation Plan (NTP). The NTP vision is to create an infrastructure and clear a path for achieving the objectives of the Vision 2030.

NTP consists of 8 themes, and among the various themes, one of the most important ones is the "health care services." NTP not only focuses on quality health services, but also prioritizes the digital records growth to $70 \%$ by 2020 . The government is thus focusing on implementing the electronic database of the patient's medical history timely and effectively. NTP also prioritizes the timely quality of health access to all the citizens and creates 


\section{S. Albahli et al. / Advances in Science, Technology and Engineering Systems Journal Vol. 5, No. 1, 40-47 (2020)}

awareness about patient rights. It also provides a blueprint for effective health services to the masses taking advantage of the electronic services. It is thus an interesting direction to study the progress made by NTP in the health sector. Furthermore, we are also interested in NTP tackling the health issues compared to the European nations. These parameters thus motivated us to analyze a number of variables related to the KSA health in general and Al Qassim province in particular.

Therefore, in this paper, the healthcare system of KSA in general and $\mathrm{Al}$ Qassim, in particular, is analyzed with respect to global European standards. In practice, the creation of an infrastructure for storing and seamless sharing of health data between different entities is studied. This helped in performing an in-depth analysis of current practices with respect to the health data, as well as finding the similarities between NTP 2020 and Health 2020 (European Union). Finally, a multi-level blockchain eHealth system is proposed to provide seamless electronic health records to patients. Lastly, the current practices being employed in Al Qassim province are also analyzed. In the study, it was found that private hospitals give online access to medical reports of their patients besides allowing them to manage their appointments. On the other hand, access to the government hospitals' medical records is minimal, especially in $\mathrm{Al}$ Qassim province.

The rest of the paper is organized as follows: Section 2 presents the related work. Section 3 explains NTP 2020 and Euro Health. Section 4 presents the Smart Health Framework. Section 5 discusses different perspectives. Section 6 presents use cases and analysis of current practices, and Section 7 concludes the paper.

\section{Related Work and Open Challenges}

The term "Smart City" has gained considerable popularity in recent years. The concept of the smart city refers to the urban infrastructure utilizing the latest Information and Communication Technologies (ICT) for citizen welfare. In general, a smart city should have three properties: Firstly, it allows the collection of data related to various urban activities and provides a flexible system to analyze and process it. Secondly, it can find and support immediate solutions using sensors and processing units interconnected through different media. Thirdly, it combines the information from multiple modalities and thus helps in decision making, thereby, adopting an information route between the municipality and different urban communities $[6,7]$.

In [8] the authors have analyzed the architectural design of IBM's Intelligent Operating System (IOS) as a smart solution for cities to combine different departments.

As noted by [9], the 19th century could be regarded as an era of empires whereas the 20th century was a century of nation-states. However, the current century would be an era of cities. The cities have become a focal point of innovation. However, most of the cities are being managed under different domains with separate entities.
A number of smart cities are planned in Saudi Arabia as well. They include King Abdullah Economic City in Rabigh with an investment of 100 Billion Saudi Arabian Riyal (SAR); Knowledge Economic City in Medina with a cost of 25 Billion SAR focusing on Education and Media supplemented by real estate and tourism; Prince Abdul Aziz Bin Mousaed Economic City in Hail with an investment of 30 Billion SAR focusing on agri-business, building materials and logistics; and Jazan Economic City with an investment of 100 Billion SAR with a focus on heavy industry projects.

The Kingdom of Saudi Arabia is the largest ICT market in the Middle East by both capital volume and spending [10]. According to [11], a smart city involves the use of data along with processes that respond to the data. Most of the efforts go in topdown fashion without involving the general public. Moreover, it fails to concentrate on the citizens' needs and views them as passive consumers of city services and mere generators of data. On the other hand, citizens should be involved in the co-creation process of different services. Similarly, they have discussed a smart city project for Milton Keynes, the United Kingdom whereby smart innovative solutions are being developed keeping in view its expected growth until 2026. One of the key components of this city is its "data hub" comprising of data from energy and water consumption along with the transport.

In [12], the authors presented an integrated framework to better understand the smart cities. They identified eight key factors for the smart city concept: management \& organization, technology, governance, policy context, people \& communities, economy, built infrastructure along with the natural environment.

Furthermore, Health Level Seven (HL7) is an American National Standards Institute accredited Standard Developing Organization (SDO) working in the healthcare industry [13]. It deals with diverse issues revolving patients such as patient administration, patient care, results operating, etc. The standards are used for information exchange within a healthcare organization as well as between various healthcare organizations. Moreover, HL7 contains model requirements for Personal and Electronic Health Records (PHR / EHR).

In [14] the authors have discussed how smart city ICT could enable effective healthcare provision along with lowering the associated costs. They discussed how the data collected from the mobile devices could be used for personal healthcare as well as healthcare of an entire smart city [14]. Users normally search for different options for nutrition, fitness, doctors, etc. Smart cities could also employ embedded technologies such as ambient sensors to fulfill the health needs of its citizens.

Increasing pressures and demands on health systems call for a change in the way of organizing and managing the delivery of health services: The quality of service expected from the hospitals and the increasing number of patients in KSA hospitals calls for a productive change in the procedures of managing and delivering 


\section{S. Albahli et al. / Advances in Science, Technology and Engineering Systems Journal Vol. 5, No. 1, $40-47$ (2020)}

health services. These include but are not limited to the following procedures:

- Digitization, integration of care services and including new services

- Introduction of patient-centered health care rather than doctor-centered health care

- Treating patients as customers to the hospital

- Managing shortages and distribution of health resources

- Remote accessibility of patients' records

The usage of electronic applications and tailored solutions are infesting our daily lives and helping to tackle many challenges of society. Therefore, it is believed that integrated eHealth is a part of the solution if the current technologies are used for improving health services.

Health 2020 [15] is a framework adopted by the European Union to merge the governmental approach with the societal one to improve the overall health conditions of populations. It also helps in removing the inequalities in health services, envision public health and promote people-centered health systems that are implementable, sustainable and deliver high-quality services to the people. As such, Health 2020 guides the European infrastructures and governments to have an eHealth vision, sets a strong strategic direction and provides suggestions for improving health services. It also addresses the inequalities found in health services and lays a future path.

Mobile and communication devices have penetrated the societies and many masses are using these devices excessively than ever before [15]. As an example, every person in Europe uses the Internet at least once or twice daily [14]. An interesting pattern of search has been found and which motivates the development of eHealth services. In [16] the authors showed that half the population of Europe with Internet access is using it for searching health-related tips and information. In [17], it is found that eHealth has enabled the patients to talk to doctors and medical specialists who are being available online. Therefore, it is believed that a similar trend lays ahead and is becoming a necessity in the KSA in general. As such, effective and efficient eHealth services are the need of the day. In this context, the Vision 2030 and the National Transformation Program (NTP) 2020 [5] motivates the investment in health services, focus on eHealth by increasing spending in this area. It also motivates the increase of private-sector spending from 25 percent to 35 percent. The government wishes to increase the facilitation for Saudi nurses and supporting staff to 150 per 100000 from 70.2 .

To meet the policy implementation requirements, the KSA needs to push IT infrastructure and integrating the eHealth paradigm in its primary health care package, improving health care governance, improving patient safety and quality of service, and integrating data analytics in eHealth.

The current KSA vision supports the digital healthcare paradigm introduction for masses and innovations as a key for realizing the KSA Vision 2030. The eHealth integration and IT solutions will help in elevating the quality of healthcare and providing a better quality of service [18].

Based on the flexibility it provides, many health systems have relied upon the cloud to store electronic health records. This has multiple benefits: It enables seamless sharing of health records among small clinics to hospitals but also takes advantage of the reliable storage facilities of IT giants like Amazon and Google. This thus reduces the burden of the hospitals in arranging and maintaining the data centers locally thereby reducing costs and focusing on primary service of health care only [19]. Figure 1 shows the generic model for integrating eHealth for the reforms process in a society [20]. The following are the challenges for the eHealth paradigm [21].

- Lack of technical expertise and computer skills.

- Failure of adoption Health Information Services

- Human and cultural barriers

- The meaningful sharing and combining of health records data between heterogeneous systems.

- Medication safety

- Financial barriers

- Security and Privacy

\section{Healthcare Initiatives: NTP 2020 and Euro 2020}

Euro Health 2020 is the framework of European policy that supports government and society in the interest of health and wellbeing [15]. Fifty-three countries in the European Region endorsed a new evidence-based concept of health policy and value for the region which was termed as the "Health 2020". Health 2020 focuses on improving health for all and reducing health inequality through improved leadership and management for health. The focus is on today's serious health problems. Euro Health 2020 identifies a number of priority areas focusing on asset development and sustainability in communities, empowerment, and enabling healthy environments. Euro 2020 also presents detailed guidelines for promoting public health services and improving the current health systems. Health 2020 was approved in two forms: a European policy framework that supports actions in government and society for the health and well-being of politicians and policymakers, and a health policy framework and strategy 2020 that provides more detailed details. The application of Health 2020 in related countries is currently a top priority for the Region.

Vision 2030 consists of 96 objectives, with the number of Key Performance Indicators (KPIs). Different KSA governmental bodies cooperate to achieve these objectives. A number of initiatives, jointly developed with organizational bodies with the support of the strong will of the current ruling setup are transforming many sectors in KSA. The vision also includes private, and non-profit organizations to provide input support for the new vision. The Council of Economic and Development affairs constituted an integrative governance model to achieve the objectives, and thus realize the vision [5]. For the capacity building and for achieving Vision 2030, the KSA setup has launched NTP, which involves the 24 government agencies. The 


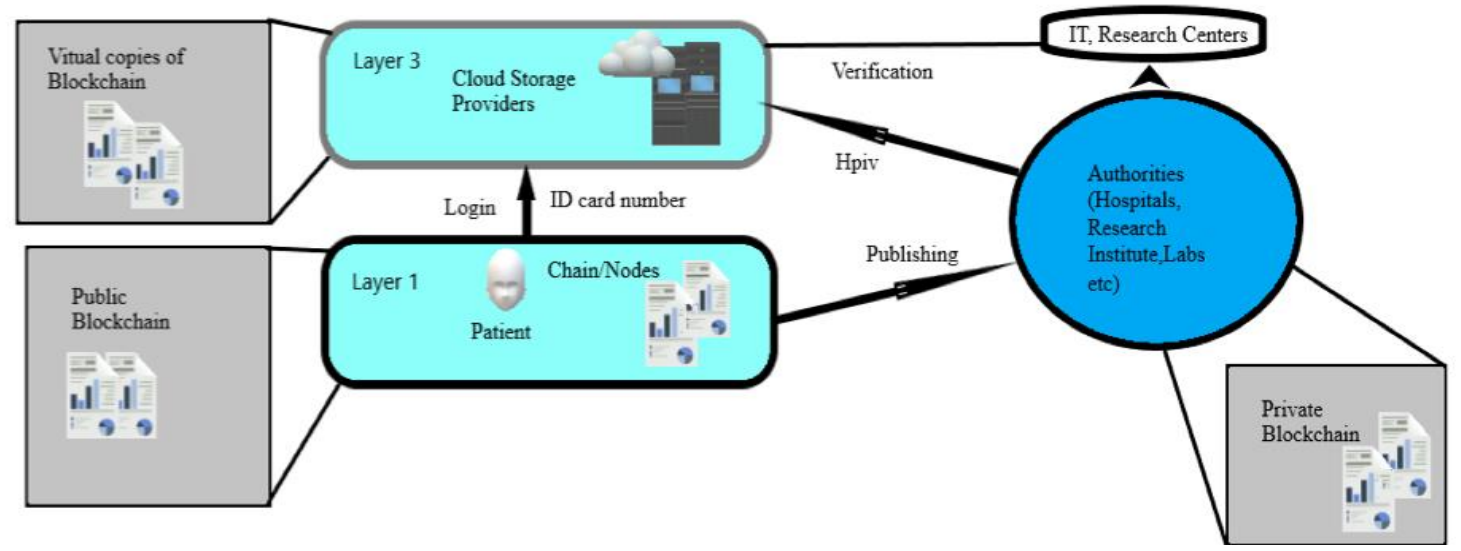

Figure 1: The generic model integrating the eHealth for reforms process in a society (Adopted from [20])

NTP vision is to create an infrastructure and clear a path for achieving the objectives of the vision 2030. NTP consists of eight directions, which are termed as "themes". Among the eight themes, one of the most important ones is the health care services.

The importance the KSA government gives to health is clear from the NTP 2020 document [5]. In NTP, the health theme is the first and given top priority. In fact, the health restructuring is considered a key element to achieve a vibrant society. The NTP aims to promote a new model of health care that has a focus on disease prevention, and then improving health awareness. The NTP has key parameters to improve access to health. For this, NTP advocates the optimal coverage, equitable geographical health facilities distribution, and health facilities expansion through e-health digital solutions.

\subsection{NTP health objectives}

The NTP health objectives which are directly or indirectly in line with the Euro Health 2020 are as follows:

\section{Access facilitation to Health Services}

2. Health services quality and efficiency improvement

The health theme of the NTP 2020 and Euro Health 2020 has a number of similarities. The reason is that Euro Health 2020 sets standards and benchmarks for all the world countries and societies. Many countries take help from Euro 2020 for improving the health system in their countries. The major differences are in implementation techniques and methods for the realization of the key points. In a comparative study, it was discovered that all the points of NTP are in line with Euro Health directly or indirectly.

\section{Smart Health Framework: Multi-Layer Blockchain}

The current interoperability challenges in the health care sector can be solved with the help of blockchain. Blockchain allows individuals, health care providers, and researchers to share health data in a secure way. In this paper, an e-Health system architecture is proposed that will not only improve the security of data but also facilitate the IT sector and research community. A blockchain-based access-control manager is proposed to keep store health records that would share a nationwide interoperability roadmap. Interoperability is a cardinal factor of any system that deals with the Precision Medicine Initiative (PMI) and the PatientCentered Outcomes Research (PCOR).

Blockchain-based IT infrastructure has come forward to increases the potential development of precision medicine and advance medical research. Blockchain is digitized and decentralized technology that forms a shared database to store data. It has three main components: a distributed network, a shared ledger, and digital transactions. The first layer consists of participant nodes that store an identical copy and helps collectively towards certifying and validating digital transactions for the network. The second layer allows records to be stored. The members who run the algorithm to add new ones verify these transactions. If the majority of participants agree with the added transaction, it approves the validity of that transaction. No single member can permit or alter the data once it has been added.

A blockchain is a digitized, decentralized, distributed public ledger that acts as a shared and synchronized database that records cryptocurrency transactions. While blockchains are essentially decentralized databases, there is no primary ownership of the data $[22,23]$. Through collaboration, users decide which data are added to the blockchain while ensuring that identical copies of the data are received and automatically updated [24].

Blockchain removes the need for a third-party and saves the financial mechanism. Finally, blockchain technology can permit the world's poorest person to access a unilateral and a corrupt-free system of wealth [25].

The digitized data is authenticated and secured with the encryption method [26]. Blocks are stored in a linear, chronological order. There are currently no open standards or implementations of blockchain that utilize this approach, but the research supports the feasibility of the proposed solution.

The proposed method has three layers:

1. The constraint nodes (IoT, Sensors, Gateways, Patients)

2. Authorities (Hospitals, Research Institute, Labs, etc.)

3. EHR layer (Cloud Providers, Storage Banks) 


\section{S. Albahli et al. / Advances in Science, Technology and Engineering Systems Journal Vol. 5, No. 1, 40-47 (2020)}

A multi-layer framework based on blockchain in IoT infrastructure has been presented. The Elliptical curve approach for Cryptography was used instead of a simple technique to make it more secure and reliable. The authentication and key generation processes moved from the Internet of things (IoT) to their respective gateways resulting in reducing the computational burden. Patient virtual blocks are introduced in the proposed model that will hide the original identity of the patient to make it more secure and efficient.

The authors used MIRACL which is a C library that allows secure communication in the blockchain. Multi-precision integers, datatypes, and cryptography are implemented through this library. Any blockchain for health care needs to be public that facilitates the research centers to promote research of the medical domain. The blockchain data contains health records, images, reports, medications, etc. An index is proposed for the information stored in the blockchain, which is like the card. That card has the metadata that has the exact location of the data of respective patients. The transactions are done in encrypted blocks. The health blockchain stores all the data related to medical history. Various metadata handle all the frequently used queries. The data can be retrieved from the hospital entities, laboratories, sensors, or from the patient itself.

All the medical data against each patient will be stored in the virtual blockchain passes towards research centers. It is an important feature of the proposed model. It includes many applications including data mining, text classification, text mining, real-time analysis, batch queries, machine learning [27]. It would be a beneficial tool for the research community and doctors. It would be helpful in finding optimal solutions based on genetics makers and real-time analysis. It will connect the whole community into one platform. The important queries can be answered through a proper and authentic channel.

The following scenarios for the proposed model as shown in Figure 1 are proposed:

1. Data has been collected by the patient from chain/nodes to create EHR.

2. EHR has been accessed by the patient or provider (authority).

3. Now, the patient can add more blocks due to his visit to the provider as seen in Figure 2.

Figure 2 shows the first layer uses public blockchain technology as it covers the new entry into different blocks. These new entries can be from patients, hospitals and sensors. They have to complete their initial registration. The block parameters include patient ID, ID card number, services, the hash of the earlier block. After the registration process, sensors can be accessed through private keys. The user itself has the public key used to create a digitized signature to accept the access permission. A digital signature is imposed for the verification of documents including lab tests, MRIs and prescriptions, etc.
The second layer includes the private blockchain infrastructure. The health record of patients is created by healthcare institutes. Let's say, a patient visited any hospital, the permitted providers created the block in the system. The data will be into the cloud storage provider for the presence of a unified record. The provider has to restrict the right to avoid EHRs abuse. Every patient holds a single block when a visit is made to any hospital containing the information of time and date. The next time when he visits any other clinic, the data is being added to any other chain/node. On the cloud side, they are attached in a linear order to maintain history and current progress. This helps in saving time.

Moreover, the patient would have the authority to choose a virtual identity. He/She can use random value $M$ as a public key by incorporating public key as $H_{\text {pub }}=M$. $H$ and private key as $H_{\text {priv }}$ $=$ M.H.S. In the second layer, the patient is authenticated by using its private key and then the cloud service provider decrypting the data by using its public key. That's how identity can be secured. Every individual owns his chain block in the blockchain, triggered by other health and IT sectors. The encrypted data is further passed for processing and storage. A notification is sent to the patient when new data is added in the blockchain. Similarly, the patient itself can store data into blocks of blockchain with digital signatures for authorization purposes.

The user enjoys full access to his data and control of sharing his data. There would be a set of permissions for who can write and update data in his block. The permissions would be flexible

instead of "all or none". The user can choose the control of his block, who can write data, time period of excess and types of data that can be shared. The user can change permission once they are chosen. These steps allow transparency in society.

When a health care provider gets the permission of access from the user, he can query the blockchain and use the ID for authentication. They can use a customized application to analyze the data. That is how a user is a single entity having control over his data and the power to grant permission for collaboration and communication during disease treatment. Digitally signed transactions combined with decentralized nature makes blockchain a secure network by having control over the network's resources.

Blockchain offers many benefits to the IT health sector. It is open-source software, developed by experts. It is transparent, reliable, responds faster against speedy changes that couldn't be chased by using closed and patented software. Open-source software offers wide innovation and the possibility of choosing options according to needs and demands. Blockchain technology runs on commodity hardware, provides low-cost high computation. They built by various vendors based on open standards. They are based on the best practices in the market because they are easy to implement and discard the complex point to point data integration. Patients, health care entities, the research community can share their accurate data in a timely manner. This 


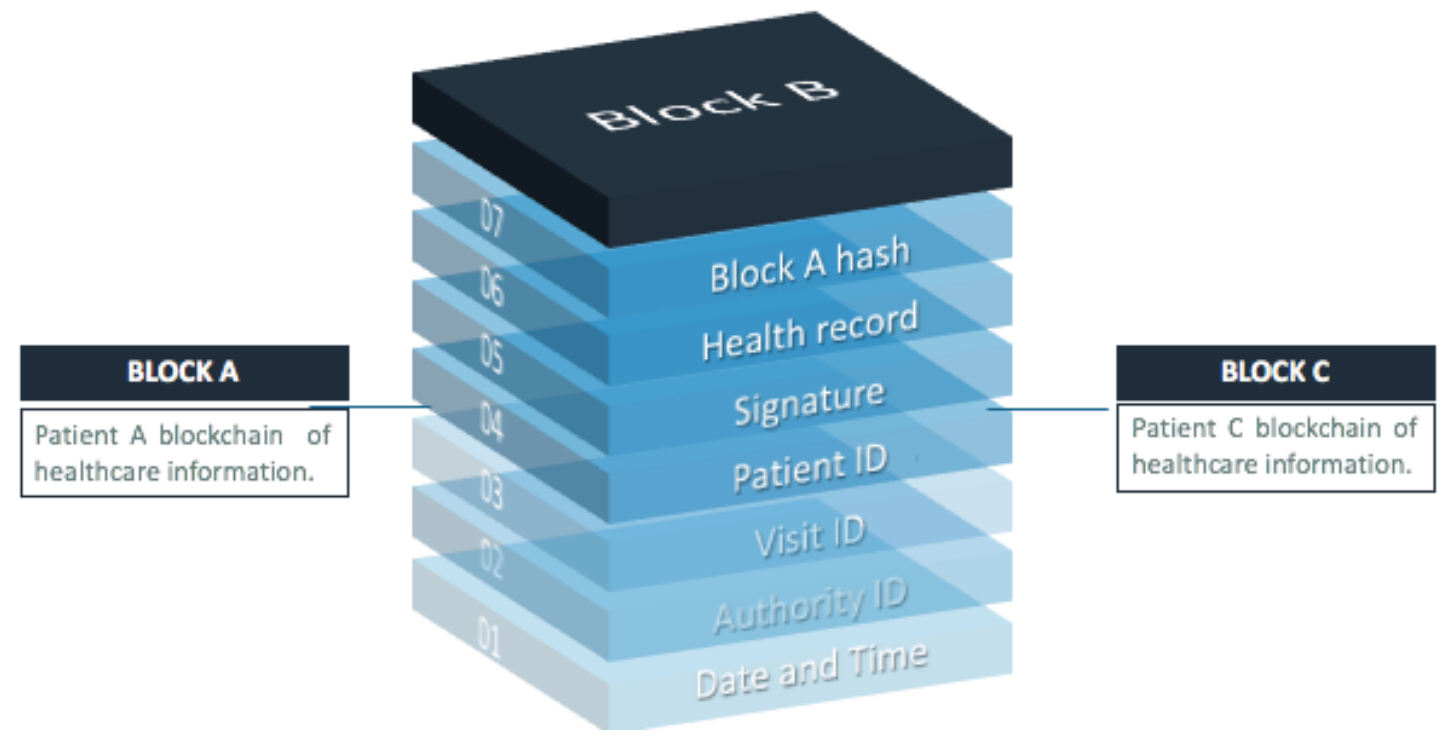

Figure 2: Patient health records in the blockchain. Each patient has one blockchain of healthcare alone

technology has been accepted and analyzed as a secure and efficient system across many private and government agencies. However, comparing cloud-based solutions with blockchain, the blockchain has gained many benefits with regards to security, flexibility, cost, and reliability. Thus, the cost and security of blockchain technology seem to be a much better solution to cloud cost and security concerns. With these solutions, for example, no third parties can access data due to each node only stores encrypted medical records so that providers and patients can help keep their own information safe from prying eyes.

The developed framework contains three layers as described earlier. Taking advantage of the blockchain technology with the cloud computing offered by [7], the proposed framework could store and share seamless health data between different entities, reducing the cost of securing and protecting patients' records. Additionally, the privacy of patients and security in the healthcare domain is preserved in order to maintain the immutability of EHRs. Thus, combining blockchain with the approach of [7] provides better performance with cryptographic adoption to make patient data secure. Moreover, the proposed framework offers immutable medical records to be an essential part of the blockchain mechanism.

\section{Discussion}

eHealth is an umbrella for connecting patients, doctors, research community and government agencies. In the context of KSA, power is ruled by monarchs. Reform is an ongoing process to improve socio-economic and political values. The government is also trying to improve the health sector through marketization and privatization. This study presents the impact of the health sector in any state, problems towards previous models and government policies of KSA. Additionally, KSA 2020 vision for the health sector is also discussed. eHealth technology benefits people in a more efficient way and it is more cost-effective.
The proposed model use interoperability objectives based on open standards to establish national technology infrastructure. An important tool to connect healthcare centers with the IT sector, the research community, and various agencies has been introduced. The purpose behind this is to improve the health and lifestyle of people in KSA. The model offers services towards analyzing the statistics and generating a report. The excess hardware capacity of multi-layer blockchain could be helpful for resource allocation, privacy, and reliability resulting in a faster solution and processing time. Multiple incentives and benefits will promote hospitals to develop solutions that allow for patient's data to be reachable across different hospitals, and not just within a single health system and hospital.

The utilization of the proposed method has enough potential to connect millions of users, health care entities, health care providers and medical researchers to share vast amounts of health data, genetics, lifestyle with guaranteed privacy and security.

\section{Use cases and analysis of current practices}

Increasing pressures and demands on health systems call for a significant change in the way of organizing and managing the delivery of health services. The quality of service expected from the Primary Health Centers (PHCs) and hospitals along with an ever-increasing number of patients in KSA hospitals calls for a productive change in the procedures of managing and delivering health services. Various doctors working in government hospitals like King Saud Hospital Unayzah, Qassim University Clinics, King Fahad Specialist Hospital Buraidah and Dr. Sulaiman AlHabib hospital, Buraydah in the private sector were consulted in order to analyze the current practices in these health centers.

These include but are not limited to the digitization of care services. It was found that almost all of the hospitals have digital patient health records. The patients are allotted an identification number during their first visit to the facility. This ID is then used 


\section{S. Albahli et al. / Advances in Science, Technology and Engineering Systems Journal Vol. 5, No. 1, 40-47 (2020)}

to manage all consultations, laboratory tests, provision of medicines, etc. This enables these centers to provide a better quality of service to the patients. Furthermore, it reduces a lot of paperwork, though it is still being maintained. However, Sulaiman Al-Habib hospital enables the patients to register online. Furthermore, the patients can book and manage appointments online besides having access to their laboratory and radiology results.

- Sulaiman Al-Habib, being the only private hospital discussed in this research, could be considered as one of the patientcentered hospitals. Everything revolves around the patients and the patients have the freedom to choose the doctors for their appointments. Moreover, patients can ask questions to the visited doctors and give ratings to the doctors as well.

- The influx of patients to secondary and tertiary care hospitals has been increased over the last few years. This pattern is more obvious in the case of Emergency and adversely affects the provision of timely care to critical patients. Therefore, many patients who are not in need of emergency treatment are being referred to the PHCs. Moreover, doctors serving in the hospitals are being asked to help the PHCs so as to manage the increased load. This has resulted in better management of seriously ill patients.

- Although most of the hospitals in KSA have digitized their records, yet there is no process to share the patients' data between different health centers. King Faisal Specialist Hospital, Riyadh has deployed a solution by Informatica which is HL7 compliant. The following are some of the common issues faced by the patients:

- The patients of government hospitals have got no access to their medical records. In the case of private centers, the data is accessible though not shared with other hospitals or pharmacies. In many cases, if a patient visits a different center, the medical tests have to be reconducted. Sometimes, patients are given CDs containing their Magnetic Resonance Imaging (MRI), X-Ray results. Even in this case, the CDs can malfunction and the patient has to revisit the medical facility.

\section{Conclusion and Future Works}

In this paper, a multi-level blockchain eHealth system is proposed to provide seamless electronic health records to patients. A detailed comparison between Saudi Arabia's National Transformation Program (NTP) and the Euro Health 2020 plan is made. It was observed that the main difference lies in the implementation techniques and methods employed for the realization of these plans. The proposed blockchain-based model is composed of constraints nodes, authorities and cloud providers. The user has complete control of his data. Moreover, the current practices being employed in Qassim, KSA has also been analyzed. It was found that the private hospitals give access to medical reports of their patients besides allowing them to manage their appointments. On the other hand, access to the government hospitals' medical records is very limited. In the future, the study can be extended to different regions of KSA.

\section{Acknowledgments}

The authors gratefully acknowledge Qassim University, represented by the Deanship of Scientific Research, on the material support for this research under the number 3578-coc2018-1-14-S during the academic year 1439 AH / 2018 AD.

\section{References}

[1] K. A. Stroetmann, "Analysis and Typology of Global eHealth Platforms - A Survey on Five Continents" Stud Health Technol Inform, 209, 162-169, 2015. https://doi:10.3233/978-1-61499-505-0-162

[2] D. Langbecker, L. J. Caffery, N. Gillespie, A. C. Smith, (2017). "Using survey methods in telehealth research: A practical guide" Journal of Telemedicine and Telecare, 23(9), 770-779, 2017. https://doi.org/10.1177/1357633X17721814

[3] E. E. Ali, L. Chew, K. Y.-L.Yap, "Evolution and current status of mhealth research: a systematic review" BMJ Innovations, 2(1), 33-40, 2016.

[4] S. A. Talesh, "Data breach, privacy, and cyber insurance: How Insurance Companies Act as "Compliance Managers" for Businesses" Law \& Social Inquiry, 43(2), 417-440, 2017. https://doi.org/10.1111//si.12303

[5] KSA Ministry (2018). "National Transformation Program | Saudi Vision 2030". Retrieved from https://vision2030.gov.sa/sites/default/files/attachments/NTP\%20English\% 20Public\%20Document_2810.pdf.

[6] C. Harrison, I. A. Donnelly, "A theory of smart cities" in 55th Annual Meeting of the International Society for the Systems Sciences (ISSS), Hull, 2011.

[7] H. F. El-Sofany, K. F. Al-Otaibi, M. S. Alsanea, "Data exchange model of patient records in the Kingdom of Saudi Arabia using cloud computing" International Journal of Innovation, Management, and $\begin{array}{llll}\text { Technology, } & 3(5), & 580-589, & \end{array}$ https://doi.org/10.7763/IJIMT.2012.V3.301

[8] L. Zhuhadar, E. Thrasher, S. Marklin, P. O. Pablos, "The next wave of innovation - Review of smart cities intelligent operation systems" Computers in Human Behavior, 66 (2017) 273-281, 2017 https://doi.org/10.1016/j.chb.2016.09.030

[9] A. Bhowmick, E. Francellino, L. Glehn, R. Loredo, P. Nesbitt, S. W. Yu, "IBM intelligent operations center for smarter cities". IBM International Technical Support Organization.

[10] Kylie Wansink "Saudi Arabia - Telecoms, Mobile and Broadband - Statistics and Analyses". Retrieved from https://www.budde.com.au/Research/SaudiArabia-Telecoms-Mobile-and-Broadband-Statistics-and-Analyses (6 May 2019)

[11] D. Gooch, A. Wolff, G. Kortuem, R. Brown, "Reimagining the role of citizens in Smart City projects" in ACM International Joint Conference on Pervasive and Ubiquitous Computing and ACM International Symposium on Wearable Computers, Osaka, Japan, 2015. https://doi.org/10.1145/2800835.2801622

[12] H. Chourabi, T. Nam, S. Walker, J. R. Gil-Garcia, S. Mellouli, K. Nahon, T. A. Pardo, H. J. Scholl, "Understanding Smart Cities: An Integrative Framework" in 45th Hawaii International Conference on System Sciences, Maui, HI, USA, 2012. https://doi.org/10.1109/HICSS.2012.615

[13] D. H. Gustafson, J. C. Wyatt, "Evaluation of eHealth systems and services" BMJ, 328, 1150, 2014. https://doi.org/10.1136/bmj.328.7449.1150

[14] D. J. Cook, G. Sprint, G. E. Duncan, R. Fritz, "Using Smart City Technology to Make Healthcare Smarter" Proceedings of the IEEE, 106(4), 708-722, 2018. https://doi.org/10.1109/JPROC.2017.2787688

[15] Regional officer for EU, "Health 2020: the European policy for health and well-being," World Health Organization, Sep. 2019.

[16] C. H. Lee, K.-H. Kim, "Implementation of IoT system using block chain with authentication and data protection" in IEEE International Conference on Information Networking (ICOIN), Chiang Mai, Thailand, 2018. https://doi.org/10.1109/ICOIN.2018.8343261

[17] C. O. Rolim, F. L. Koch, C. B. Westphall, J. Werner, A. Fracalossi, G. S. Salvador, "A Cloud Computing Solution for Patient's Data Collection in Health Care Institutions," in IEEE Second International Conference on 
eHealth, Telemedicine, and Social Medicine, St. Maarten, Netherlands Antilles, 2010. https://doi.org/10.1109/eTELEMED.2010.19

[18] A. F. Hussein, N. ArunKumar, G. Ramirez-Gonzalez, E. Abdulhay, J. M. R. S. Tavares, V. H. C. de Albuquerque, "A medical records managing and securing blockchain based system supported by a Genetic Algorithm and Discrete Wavelet Transform" Cognitive Systems Research, 52, 1-11, 2018. https://doi.org/10.1016/j.cogsys.2018.05.004

[19] M. Moran, "Understanding the welfare state: the case of health care" The British Journal of Politics and International Relations, 2(2): 135-160, 2000. https://doi.org/10.1111/1467-856X.00031

[20] T. Dey, S. Jaiswal, S. Sunderkrishnan, N. Katre, "HealthSense: A medical use case of Internet of Things and blockchain" in IEEE International Conference on Intelligent Sustainable Systems (ICISS), Palladam, India, 2017. https://doi.org/10.1109/ISS1.2017.8389459

[21] O. Alphand, M. Amoretti, T. Claeys, S. Dall 'Asta, A. Duda, G. Ferrari, F. Rousseau, B. Tourancheau, L. Veltri, F. Zanichelli, "IoTChain: A blockchain security architecture for the Internet of Things" in IEEE Wireless Communications and Networking Conference (WCNC), Barcelona, Spain, 2018. https://doi.org/10.1109/wcnc.2018.8377385

[22] V. A. Patel, "A framework for secure and decentralized sharing of medical imaging data via blockchain consensus" Health Informatics J., 25(4), 1398$1411,2018$. https://doi.org/10.1177/1460458218769699

[23] M. Conoscenti, A. Vetrò, and J. C. D Martin, "Blockchain for the Internet of Things: A systematic literature review" in IEEE/ACS 13th International Conference of Computer Systems and Applications (AICCSA), Agadir, Morocco, 2017. https://doi.org/10.1109/AICCSA.2016.7945805

[24] W. J. Gordon, C. Catalini, "Blockchain Technology for Healthcare: Blockchain Technology for Healthcare: Facilitating the Transition to Patient-Driven Interoperability" Computational and Structural Biotechnology Journal, 16, 224-230, 2018. https://doi.org/10.1016/j.csbj.2018.06.003

[25] H. Kaur, M. A. Alam, R. Jameel, A. K. Mourya, V. Chang, “A proposed solution and future direction for blockchain-based heterogeneous medicare data in cloud environment" Journal of Medical Systems, 42(8), 156, 2018. https://doi.org/10.1007/s10916-018-1007-5

[26] T. K. Mackey, G. Nayyar, "A review of existing and emerging digital technologies to combat the global trade in fake medicines" Expert Opinion on Drug Safety, 16(5), 587-602, 2017. https://doi.org/10.1080/14740338.2017.1313227

[27] Albahli, Saleh. "A Deep Ensemble Learning Method for Effort-Aware JustIn-Time Defect Prediction." Future Internet 11, no. 12 (2019): 246. 